\title{
Outbreaks, persistence, and high mortality rates of multiresistant Pseudomonas aeruginosa infections in a hospital with AIDS-predominant admissions
}

\section{Authors}

Marisa Zenaide Ribeiro Gomes Carolina Romero Machado ${ }^{2}$ Magda de Souza da Conceição² Jois Alves Ortega ${ }^{3}$ Sonia Maria Ferraz M Neves Maria Cristina da Silva Lourenç $0^{5}$

Marise Dutra Asensi ${ }^{6}$

${ }^{1} \mathrm{MD}$, MSc, PhD; Assistant Researcher, Instituto Oswaldo Cruz, Fundação Oswaldo Cruz (IOC/ FIOCRUZ), RJ, Brazil; Visiting Scientist, University of Texas, MD Anderson Cancer Center, USA ${ }^{2} \mathrm{MD}$; Infectious Diseases Physician, Instituto de Pesquisa Clínica Evandro Chagas/FIOCRUZ, RJ, Brazil ${ }^{3} \mathrm{MD}, \mathrm{MSc}$; Infectious Diseases Physician, Instituto de Pesquisa Clínica Evandro Chagas/FIOCRUZ, RJ, Brazil

${ }^{4} \mathrm{RN}, \mathrm{MSc}, \mathrm{PhD} ;$ Chairman of Hospital Infection Control Committee, Instituto de Pesquisa Clínica Evandro Chagas/FIOCRUZ RJ, Brazil

${ }^{5} \mathrm{MSc}$; Chairman of Laboratory of Bacteriology Instituto de Pesquis Clica Eve Intito de Pesqua Clinica Ering RJ, Brazil

${ }^{6} \mathrm{PhD}$; Chairman of Nosocomial Infection Research Laboratory, IOC/FIOCRUZ, R], Brazil

Submitted on: 12/01/2010 Approved on: 03/15/2011

Correspondence to: Marisa Zenaide Ribeiro Gomes Laboratório de Pesquisa em Infecção Hospitalar Instituto Oswaldo Cruz Fundação Oswaldo Cruz Avenida Brasil 4365, Pavilhão Rocha Lima - S319 Manguinhos,

21040-900, Rio de Janeiro, RJ

Brazil

Phone: 5521 2598-4277,

extension 319

Fax: 5521 3322-0613

marisargomes@ioc.fiocruz.br

mrgomes@mdanderson.org

Financial Support: MZRG received finantial support from FIOCRUZ and Fundação Carlos Chagas Filho de Amparo à Pesquisa do Estado do Rio de Janeiro (FAPERJ), TEC

TEC program and APQ5,

E-26/110.440/2008.

We declare no conflict of interest.

(2) 2011 Elsevier Editora Ltda. All rights reserved.

\begin{abstract}
Introduction: Authors have reported increased incidence of multiresistant Pseudomonas aeruginosa (MR-PA) infections worldwide over the last decade. Researchers have proposed multifaceted approaches to control MR-PA infections, but none have been reported in the acquired immunodeficiency syndrome (AIDS) setting. Objective and Methods: Herein we report the impact of a multifaceted intervention for controlling MR-PA over five years in a hospital with AIDS-predominant admissions and describe the clinical characteristics of MR-PA infection in our patient population. The clinical outcomes of infected patients and molecular characteristics of the isolated strains were used as tools for controlling MR-PA infection rates. Results: Significant temporary decrease of new infections was achieved after intervention, although a high level of diagnostic suspicion of nosocomial infection was maintained. We obtained 35 P. aeruginosa isolates with multiresistant profiles from 13 infected and 3 colonized patients and 2 environmental samples. Most of the patients (94\%) were immunocompromised with AIDS ( $\mathrm{n}=10)$ or HTLV-1 infections $(n=5)$. Of the followed patients, $67 \%$ had persistent and/or recurrent infections, and 92\% died. We observed differences in the antibiotic-resistance pattern of MR-PA infection/colonization during two outbreaks, although the genetic profiles of the tested strains were identical. Conclusions: Therefore, we concluded that early multidisciplinary interventions are essential for reducing the burden caused by this microorganism in patients with AIDS. Prolonged or suppressive antibiotic-based therapy should be considered for MR-PA infections in patients with AIDS because of the persistence characteristic of MR-PA in these patients.
\end{abstract}

Keywords: Pseudomonas aeruginosa; disease outbreaks; infection control; molecular epidemiology; acquired immunodeficiency syndrome.

\section{INTRODUCTION}

Multiresistant Pseudomonas aeruginosa (MR-PA) is one of the leading pathogens that cause nosocomial infections and outbreaks worldwide. ${ }^{1-8}$ These infections are usually associated with high mortality rates. ${ }^{1-6}$ Also, in patients with human immunodeficiency virus (HIV) or acquired immunodeficiency syndrome (AIDS), authors have described persistent and recurrent infections caused by non-MR-PA strains. ${ }^{9,10}$ Pulsed-field gel electrophoresis (PFGE) is the recommended technique for determining the genotypes of nosocomial pathogens. ${ }^{11}$ Researchers have used it in several studies to confirm the similarity of $P$. aeruginosa strains, suggesting the importance of cross-colonization, ${ }^{11,12}$ and as a tool to control nosocomial infections. ${ }^{3,12}$ Multifaceted strate- gies using molecular epidemiology approach for controlling MR-PA infection outbreaks in immunocompromised patients, especially those with cancer or admitted to intensive care units (ICUs), ${ }^{3,5}$ have been adopted but none were described in patients with AIDS. In this report, we describe the surveillance and intervention measures used for controlling MR-PA infections over five years in an infectious disease hospital in Rio de Janeiro, Brazil, in which the majority of admitted patients have AIDS. We also describe the clinical evolution of patients with MR-PA infections or colonization and the antimicrobial susceptibility profiles and molecular characteristics of MR-PA strains recovered from the patients and their environments. Increased knowledge of the epidemiology and clinical presentations 
of MR-PA infection in this patient population may improve early interventions.

\section{MATERIAL AND METHODS}

\section{Institution and surveillance program}

A prospective program for controlling nosocomial infections, which used an adaptation of methodologies developed by the Centers for Disease Control and Prevention, was initiated in April 2002 in a 26-bed (including 2-4 ICU beds) hospital from Fundação Oswaldo Cruz (FIOCRUZ). ${ }^{13-15}$ The adaptation concerned the application of surveillance in the entire hospital and used to identify nosocomial infections in patients with AIDS considered to be acquired at the hospital. ${ }^{16}$ Infections were considered nosocomial if they first appeared $48 \mathrm{~h}$ after admission. Infections that were likely incubating before hospital admission were not considered nosocomial. All clinical and surveillance cultures obtained from every admitted patient were observed daily for early identification and control of multiresistant pathogens from April 2002 to February 2007. Three antibiotic susceptibility patterns of MR-PA were considered: I) multidrug-resistant $P$. aeruginosa (MDR-PA) was indicated by strains resistant to carbapenems and, concomitantly, at least two other classes of antipseudomonal antibiotics: penicillins, cephalosporins, monobactams, aminoglycosides, and fluoroquinolones; II) extensive drug-resistant $P$. aeruginosa (XDR-PA) was indicated by strains resistant to all classes of antipseudomonas antibiotics with the exception of polymyxin B; III) carbapenem-resistant $P$. aeruginosa (CR-PA) was indicated by strains resistant only to carbapenems. All the patients' medical records were reviewed to confirm the prospectively collected data.

During MR-PA outbreaks, in addition to clinical cultures a differential surveillance was performed, which included rectal swab samples for MR-PA detection obtained from patients hospitalized in the same room or unit whenever an MR-PA isolate was identified. Cultures of clinical surface samples were obtained during the first outbreak in the ICU where the majority of the patients with acquired MR-PA were hospitalized. These samples were collected before cleaning from furniture, equipment, sinks, ventilation ducts, air conditioning intake grills, air conditioning filters, compressed air tubes, patients' nebulizers and respiratory circuits with sterile cotton-tipped swabs moistened with transport media. Water from air conditioner compressors and humidifiers was also sampled. During the second outbreak, in November 2006, the following samples were collected from three areas of the ICU and from two wards as part of another research protocol also approved by the institutional ethics com- mittee: $250 \mathrm{~L}$ of air samples taken using an MAS-100 air sampler (Merck \& Co., Inc., Whitehouse Station, NJ, USA) at a distance of 1.5-2.0 m from the patients beds and $1.5 \mathrm{~m}$ from the floor, two surface samples taken in each ICU and ward area using the in print technique with RODAC plates, and samples taken from the hands (washed in a $100 \mathrm{~mL} 0.1 \%$ peptone solution in sterile bags) of personnel who dealt with MR-PA-positive patients during environmental sampling.

\section{Laboratory procedures}

Clinical samples and rectal swabs were inoculated onto MacConkey agar (Merck \& Co., Inc.) and cetrimide agar (Merck \& Co., Inc.) plates. Environmental surface swabs were plated on sheep blood agar. Cetrimide agar, MacConkey agar, and salt mannitol agar (Merck \& Co., Inc.) were used for air sampling. The plates were incubated at $37^{\circ} \mathrm{C}$ for $48 \mathrm{~h}$. Colonies that contained Gram-negative rods were subcultured onto blood and MacConkey agar plates and incubated for $24 \mathrm{~h}$. All oxidase-positive bacilli and non-lactose fermenting growths were identified as $P$. aeruginosa using conventional biochemical tests.

The antibiotic susceptibility of the isolates was tested using the disk-diffusion method according to Clinical and Laboratory Standards Institute recommendations. ${ }^{17}$ Susceptibility to polymyxin B was verified using the E-test. Resistant strains included those with intermediate susceptibility. Screening of the strains for metallo- $\beta$-lactamase (MBL) production using a ceftazidime disk in the presence of 2-mercaptopropionic acid and polymerase chain reaction for detection of the blaSPM-1, blaIMP, and blaVIM genes were performed. ${ }^{18}$ DNA macrorestriction using Spel followed by PFGE for molecular typing of isolates was carried out as well. ${ }^{19}$ Restriction fragment profiles of the genomic DNA of MR-PA strains were compared using visual inspection and the GelCompar II software program (version 3.5; Applied Maths, Sint-Martens-Latem, Belgium) and analyzed using the Tenover criteria (1995). ${ }^{20}$

\section{Clinical characteristics of MR-PA infections}

The clinical characteristics of MR-PA infections were classified according to three categories: I) persistent infection, in which the microorganism was maintained in the same or another clinical material for at least 21 days regardless of symptoms or treatment; II) recurrent infection, in which clinical symptoms of infection reinitiated and the MR-PA was isolated in the same or another biological material after completion of treatment; and III) single infection, which was an unique infectious episode without persistence or recurrence. Treatment failure was considered in cases with any clinical deterioration or persistence of the microorganism in clinical materials resulting in a change in the antibiotic-based therapy or death. 


\section{RESULTS}

\section{Admitted patients}

HIV (850 patients) and HTLV-1 (130 patients) infections were the main clinical conditions of the admitted patients (54\% of admissions). Also, readmissions occurred in a high proportion of the patients (654 readmissions among 1,814 admissions) during the 5-year study period. An average of 35 clinical bacteriological cultures per 100 patient-days was performed in the hospital over this period.

\section{Description of outbreaks}

Figure 1 shows the monthly distribution and resistance patterns of $P$. aeruginosa isolates during the study period. The mean incidence density of MR-PA infection (using for calculation only the first MR-PA isolate obtained from each patient) was 0.53/1,000 patient-days from April 2002 to February 2007. However, it was 2.8/1,000 patient-days from November 2004 to March 2005 (first outbreak) and 2.1/1,000 patient-days from November 2006 to February 2007 (second outbreak).

The onset of the first MR-PA outbreak coincided with identification of the XDR-PA-infected index patient in the ICU in September 2004 (Figure 1). This patient came from another nearby hospital; two months before detection of the first outbreak with recovery of $15 \mathrm{MDR}-\mathrm{PA}$ and $2 \mathrm{XDR}-\mathrm{PA}$ strains from six patients. After an intervention program for controlling the MR-PA outbreak initiated in November 2004, the occurrence of MR-PA decreased significantly, with only one new CR-PA infection detected over a 1-year period (September 2005 to October 2006). During the second outbreak, which began in November 2006, we had seven XDR-PA isolates from five patients but detected no MDR-PA or CR-PA strains.

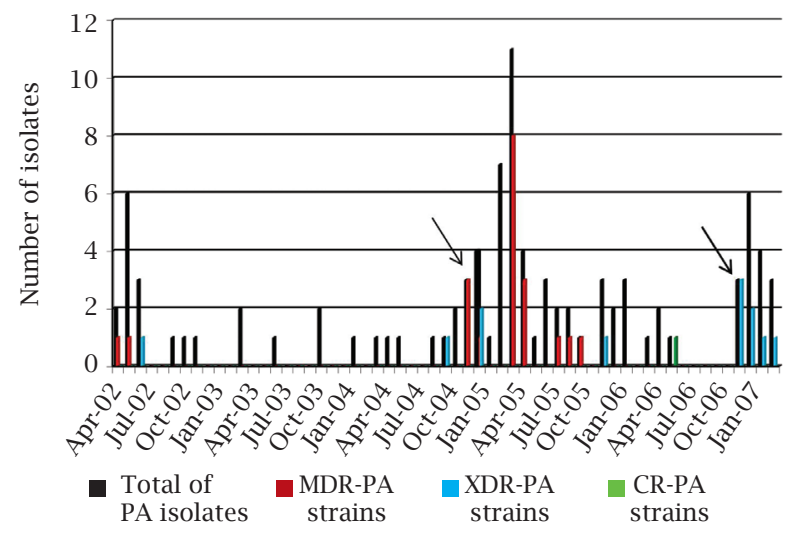

Figure 1: Epidemiologic curve showing the $P$. aeruginosa isolates obtained monthly from patients. Total $P$. aeruginosa isolates (PA, black bar) and MDR-PA (red bar), XDR-PA (blue bar), and CR-PA (green bar) isolates are shown. The arrows indicate when the infection control interventions were initiated.

\section{Culture results, clinical presentations and outcomes}

Forty-four patients had $P$. aeruginosa infections or colonization during the study. Twenty-six patients had isolates with non-CR-PA/MDR-PA/XDR-PA susceptibility patterns. Two patients had isolates with these resistance patterns detected before $24 \mathrm{~h}$ of hospital admission. One of these patients had been admitted previously during the first outbreak, but a rectal swab obtained from her was negative for MR-PA at that time. Of 16 patients in whom MR-PA developed during hospitalization in the study period, 15 were immunocompromised $(94 \%)$ with AIDS $(\mathrm{n}=10)$ or HTLV-1 $(\mathrm{n}=5)$ with urinary tract infections (UTIs) (Table 1). Thirteen patients had MR-PA infections, whereas three had MRPA colonization. Rectal swabs were positive for MR-PA in only one patient, who had MDR-PA isolates recovered from other clinical samples. Table 1 shows the age, sex, underlying and comorbid condition, length of hospital stay and admission to the ICU prior to MR-PA infection, and laboratory data for the MR-PA isolates obtained from these 16 patients.

Among six patients in whom MR-PA infection/colonization developed during the first outbreak, four were undergoing hemodialysis (patients \#5 to \#8 in Tables 1 and 2) and four (patients \#3 to \#5 and \#7) were in the ICU when they acquired it. In comparison, among the five patients in whom these infections/colonizations developed during the second outbreak, none were undergoing hemodialysis and three were in the ICU when they acquired it (Table 1). Each new MR-PA-positive patient had been hospitalized in close proximity to another previously MR-PA-positive patient in the ICU or other hospital ward. Table 2 lists the types of infections, therapies for Gram-negative bacterial infections after MR-PA isolation, MR-PA infection treatment failures, infection classifications, and deaths associated with MR-PA infection and/or colonization during the study period.

Septicemia or infections related to the use of invasive devices predominated among patients (13/19,68\%). Excluding one patient who was lost to follow-up (transferred to another hospital), 58\% ( $\mathrm{n}=7)$ of the remaining 12 infected patients had one MR-PA infection treatment failure. Also, eight patients (67\%) had persistent and/or recurrent MR-PA infections, and 11 (92\%) died despite receiving therapy and medical support (Table 2). The overall mortality rate associated with MR-PA infection during the study period was $80 \%(12 / 15)$. The average time span between obtaining the first and last clinical MR-PA-positive samples in eight patients who had persistent and/or MR-PA recurrent infections was 55.3 days (median, 29 days; range, 21-195 days). Five patients in the first outbreak $(83 \%)$ and one in the second outbreak $(20 \%)$ died. The patient who survived in the first outbreak died of recurrent MDR-PA septicemia six months after his first MR-PA infection (patient \#8). The patient who died during the second outbreak (patient \#14) had untreated XDR-PA bacteremia one month before development of XDR-PA ventilator-associated pneumonia (VAP). 
Table 1. Epidemiological data and laboratory results of patients with MR-PA infection and/or colonization*

\begin{tabular}{|c|c|c|c|c|c|c|}
\hline Patient & $\begin{array}{c}\text { Sex/ } \\
\text { age } \\
\text { (years) }\end{array}$ & $\begin{array}{c}\text { Clinical } \\
\text { condition }\end{array}$ & $\begin{array}{c}\text { Other } \\
\text { diagnoses }\end{array}$ & $\begin{array}{l}\text { Length of } \\
\text { hospital } \\
\text { stay }^{\dagger} / \\
\text { ICU } \\
\text { admission }^{\dagger}\end{array}$ & $\begin{array}{l}\text { Sample - } \\
\text { susceptibility } \\
\text { pattern, date } \\
\text { of sample }\end{array}$ & $\begin{array}{c}\text { Time span } \\
\text { between first } \\
\text { and last MR-PA } \\
\text { positive } \\
\text { cultures (days) }\end{array}$ \\
\hline 1 & $\mathrm{M} / 43$ & $\begin{array}{l}\text { HAM/TSP } \\
\text { with } \\
\text { recurrent } \\
\text { UTI }\end{array}$ & $\begin{array}{l}\text { MRSA sepsis, } \\
\text { renal failure, } \\
\text { respiratory failure }\end{array}$ & $4 / \mathrm{No}$ & $\begin{array}{l}\text { Urine: MDR-PA, } \\
\text { 05/21/02; } \\
\text { CVC tip: XDR-PA, } \\
\text { 06/10/02 }\end{array}$ & 21 \\
\hline 2 & $\mathrm{M} / 33$ & AIDS & $\begin{array}{c}\text { Cryptococcal meningitis, } \\
\text { infected dermatitis, } \\
\text { Pneumocystis carinii } \\
\text { pneumonia, Respiratory } \\
\text { failure; last CD4:406 cells/ } \mathrm{\mu L}^{\ddagger}\end{array}$ & 36/Yes & $\begin{array}{l}\text { Blood: XDR-PA, } \\
\text { (PFGE tested) } \\
\text { 09/08/04; } \\
\text { BAL: XDR-PA, } \\
\text { 09/09/04 }\end{array}$ & 2 \\
\hline 3 & $\mathrm{M} / 45$ & AIDS & $\begin{array}{c}\text { Neurologic disease, } \\
\text { DM type } 2 \text {, COPD, } \\
\text { HCV-positive, } \\
\text { Enterococcus faecalis UTI, } \\
\text { respiratory failure; last } \\
\text { CD4: } 98 \text { cells } / \mu \mathrm{L} ; \\
\text { viral load: } 19,000 \\
\text { copies } / \mathrm{mL}^{\ddagger}\end{array}$ & $5 /$ Yes & $\begin{array}{l}\text { Tracheal aspirate: } \\
\text { MDR-PA, 11/12/04; } \\
\text { BAL: MDR-PA (PFGE } \\
\text { tested), 11/29/04; } \\
\text { tracheal aspirate: } \\
\text { XDR-PA, 12/08/04; } \\
\text { vascular catheter tip: } \\
\text { XDR-PA, 12/08/04 }\end{array}$ & 27 \\
\hline 4 & $\mathrm{M} / 25$ & AIDS & $\begin{array}{c}\text { CMV esophagitis, } \\
\text { pneumothorax, respiratory } \\
\text { failure, MRSA colonization; } \\
\text { last CD4: } 324 \text { cells } / \mu L ; \\
\text { viral load: } 710,000 \text { copies/mL } \text { mL }^{\ddagger}\end{array}$ & 9/Yes & $\begin{array}{c}\text { Tracheal secretion: } \\
\text { MDR-PA (PFGE tested), } \\
\text { 11/30/04 }\end{array}$ & - \\
\hline $5 \S$ & $\mathrm{M} / 50$ & AIDS & $\begin{array}{c}\text { Febrile neutropenia, } \\
\text { disseminated Mycobacterium } \\
\text { tuberculosis, renal failure, } \\
\text { hepatic failure, pancreatitis }\end{array}$ & 20/Yes & $\begin{array}{l}\text { Tracheal secretion: } \\
\text { MDR-PA, 20,000 } \\
\text { CFU, 12/19/04 }\end{array}$ & - \\
\hline $6^{\S}$ & $\mathrm{F} / 43$ & AIDS & $\begin{array}{l}\text { Neurologic disease, } \\
\text { Salmonella and Enterococcus } \\
\text { species sepsis, MRSA CVC } \\
\text { sepsis, bronchoaspiration, } \\
\text { renal failure }\end{array}$ & 8/No & $\begin{array}{c}\text { Vascular catheter tip: } \\
\text { MDR-PA, 03/03/05; } \\
\text { urine MDR-PA, } \\
\text { 03/30/05 }\end{array}$ & 28 \\
\hline $7 \S$ & $\mathrm{M} / 59$ & Leptospirosis & $\begin{array}{c}\text { Renal failure, } \\
\text { atrial fibrillation, } \\
\text { MRSA CVC sepsis }\end{array}$ & 33/Yes & $\begin{array}{c}\text { Two vascular } \\
\text { catheter tips: MDR-PA, } \\
\text { 03/21/05; urine: } \\
\text { MDR-PA, 03/28/05 } \\
\text { and 03/31/05; } \\
\text { rectal swab: MDR-PA, } \\
\text { 04/06/05; } \\
\text { vascular catheter tip: } \\
\text { MDR-PA, 04/18/05; } \\
\text { tracheal secretion: } \\
\text { MDR-PA, 04/18/05 }\end{array}$ & 29 \\
\hline $8^{\S}$ & $\mathrm{M} / 54$ & AIDS & $\begin{array}{l}\text { Febrile neutropenia, } \\
\text { cryptococcosis, } \\
\text { high blood pressure, } \\
\text { chronic renal failure, } \\
\text { MRSA colonization }\end{array}$ & 8/No & $\begin{array}{c}\text { Blood: MDR-PA, } \\
\text { 03/10/05; } \\
\text { urine: MDR-PA, } \\
\text { 03/22/05; } \\
\text { blood: MDR-PA, } \\
\text { 9/21/05 }\end{array}$ & 195 \\
\hline
\end{tabular}


Table 1. Epidemiological data and laboratory results of patients with MR-PA infection and/or colonization*

\begin{tabular}{|c|c|c|c|c|c|c|}
\hline Patient & $\begin{array}{c}\text { Sex/ } \\
\text { age } \\
\text { (years) }\end{array}$ & $\begin{array}{l}\text { Clinical } \\
\text { condition }\end{array}$ & $\begin{array}{c}\text { Other } \\
\text { diagnoses }\end{array}$ & $\begin{array}{c}\text { Length of } \\
\text { hospital } \\
\text { stay }^{\dagger} / \\
\text { ICU } \\
\text { admission }^{\dagger}\end{array}$ & $\begin{array}{c}\text { Sample - } \\
\text { susceptibility } \\
\text { pattern, date } \\
\text { of sample }\end{array}$ & $\begin{array}{c}\text { Time span } \\
\text { between first } \\
\text { and last MR-PA } \\
\text { positive } \\
\text { cultures (days) }\end{array}$ \\
\hline 9 & $\mathrm{~F} / 60$ & $\begin{array}{l}\text { HAM/TSP } \\
\text { with } \\
\text { recurrent } \\
\text { UTI }\end{array}$ & $\begin{array}{c}\text { Cutaneous T-cell } \\
\text { lymphoma, hemorrhagic } \\
\text { stroke, high } \\
\text { blood pressure, } \\
\text { DM-type II, MDR } \\
\text { Escherichia coli UTI, } \\
\text { MRSA osteomyelitis }\end{array}$ & 53/Yes & $\begin{array}{c}\text { Urine: MDR-PA, } \\
07 / 06 / 05\end{array}$ & - \\
\hline 10 & $\mathrm{~F} / 64$ & $\begin{array}{l}\text { HAM/TSP } \\
\text { with } \\
\text { recurrent } \\
\text { UTI }\end{array}$ & $\begin{array}{l}\text { E. coli UTI, } \\
\text { hydronephrosis } \\
\text { with renal calculi, } \\
\text { decubitus ulcers } \\
\text { and osteomyelitis, } \\
\text { MRSA colonization }\end{array}$ & 9/No & $\begin{array}{c}\text { Urine: MDR-PA } \\
\text { (PFGE tested), } \\
\text { 08/15/05; } \\
\text { urine: XDR-PA, } \\
\text { 11/10/05 }\end{array}$ & 88 \\
\hline 11 & $\mathrm{~F} / 47$ & AIDS & $\begin{array}{c}\text { Hepatitis C, } \\
\text { decubitus ulcers, } \\
\text { UTI, MRSA colonization }\end{array}$ & 2"/Yes & $\begin{array}{l}\text { Urine: CR-PA, } \\
\text { 05/31/06 }\end{array}$ & - \\
\hline 12 & $\mathrm{M} / 56$ & AIDS & $\begin{array}{c}\text { Hyperparathyroidism, } \\
\text { HIV dementia, } \\
\text { CDAD; last CD4: } \\
9 \text { cells } / \mu \mathrm{L} ; \\
\text { viral load: > 100,000 } \\
\text { copies } / \mathrm{mL}^{\ddagger}\end{array}$ & $85 /$ Yes & $\begin{array}{c}\text { Blood: XDR-PA, } \\
\text { 11/16/06; } \\
\text { blood: XDR-PA } \\
\text { (PFGE tested), } \\
\text { 12/06/06 }\end{array}$ & 21 \\
\hline 13 & $\mathrm{~F} / 75$ & $\begin{array}{c}\text { HAM/TSP } \\
\text { with } \\
\text { urinary } \\
\text { sepsis }\end{array}$ & $\begin{array}{l}\text { Streptococcus } \\
\text { species sepsis }\end{array}$ & 28/No & $\begin{array}{c}\text { Urine: XDR-PA, } \\
\text { 50,000 CFU/mL } \\
\text { (PFGE tested), } \\
\text { 11/11/06 }\end{array}$ & - \\
\hline 14 & $\mathrm{M} / 31$ & AIDS & $\begin{array}{l}\text { CMV meningitis, } \\
\text { CDAD (pseudomembranous } \\
\text { colitis), MDR Klebsiella } \\
\text { pneumoniae CVC sepsis, } \\
\text { respiratory failure; } \\
\text { last CD4: } 6 \text { cells } / \mathrm{uL}^{\ddagger}\end{array}$ & 174/Yes & $\begin{array}{c}\text { Blood: XDR-PA, } \\
\text { (PFGE tested), } \\
\text { 11/24/06; } \\
\text { BAL: XDR-PA, } \\
\text { 12/26/06 }\end{array}$ & 33 \\
\hline 15 & $\mathrm{M} / 26$ & AIDS & $\begin{array}{l}\text { Febrile neutropenia, } \\
\text { MRSA sepsis }\end{array}$ & 32/No & $\begin{array}{c}\text { Urine: XDR-PA, } \\
\text { 80,000 CFU/mL, } \\
01 / 25 / 07\end{array}$ & - \\
\hline 16 & M/69 & $\begin{array}{l}\text { HAM/TSP } \\
\text { with urinary } \\
\text { sepsis }\end{array}$ & $\begin{array}{l}\text { Iatrogenic } \\
\text { pneumothorax, } \\
\text { decubitus ulcers }\end{array}$ & 31/Yes & $\begin{array}{c}\text { Urine: XDR-PA, } \\
\text { 02/02/07 }\end{array}$ & - \\
\hline
\end{tabular}

BAL, bronchoalveolar lavage; CDAD, Clostridium difficile-associated diarrhea; CFU, colony-forming unit; CMV, cytomegalovirus; COPD, chronic obstructive pulmonary disease; CVC, central vascular catheter; DM, diabetes mellitus; F, female; HAM/TSP, HLTV-I associated myelopathy/tropical spastic paraparesis; HCV, hepatitis C virus; M, male; MRSA, methicillin-resistant Staphylococcus aureus.

"MR-PA includes all resistance patterns(MDR-PA, XDR-PA, and CR-PA).

${ }^{\dagger}$ Previous to MR-PA infection or colonization.

${ }^{\ddagger} \mathrm{CD} 4$ and viral load for HIV-positive patients when available and collected less than 6 months before MR-PA infection/ colonization.

sPerformance of hemodialysis when MR-PA was acquired.

"Discharged from the study hospital 5 days before this admission. 
Table 2. Clinical presentations, antibiotic regimen, and outcomes in patients with MR-PA infection or colonization*

\begin{tabular}{|c|c|c|c|c|c|}
\hline Patient & $\begin{array}{l}\text { Type of infection/ } \\
\text { colonization }\end{array}$ & $\begin{array}{l}\text { Antibiotic } \\
\text { regimen }^{\dagger}\end{array}$ & $\begin{array}{l}\text { Treatment } \\
\text { failure }^{\ddagger}\end{array}$ & $\begin{array}{c}\text { Infection } \\
\text { classification }^{\S} \mathbf{h}\end{array}$ & $\begin{array}{l}\text { Death during } \\
\text { hospitalization }\end{array}$ \\
\hline \multirow[t]{2}{*}{1} & UTI & $\begin{array}{l}\text { Empiric cefepime } \\
\text { and guided by culture for } \\
21 \text { days, associated with } \\
\text { empiric amikacin and } \\
\text { guided by culture for } 7 \text { days }\end{array}$ & No & $\begin{array}{l}\text { Persistent } \\
\text { infection }\end{array}$ & \multirow[t]{2}{*}{ Yes" } \\
\hline & CVC sepsis & Not treated & N/A & - & \\
\hline 2 & $\begin{array}{l}\text { VAP and } \\
\text { septicemia }\end{array}$ & $\begin{array}{l}\text { The patient had received } \\
\text { cefepime and levofloxacin } \\
\text { for } 14 \text { days and was receiving } \\
\text { treatment for Pneumocystis } \\
\text { carinii pneumonia }\end{array}$ & $\mathrm{N} / \mathrm{A}$ & Single episode & Yes" \\
\hline \multirow[t]{2}{*}{3} & VAP & $\begin{array}{l}\text { Empiric levofloxacin for } 2 \text { days, } \\
\text { cefepime for } 20 \text { days, } \\
\text { empiric polymyxin B for } \\
2 \text { days, amikacin for } 16 \text { days }\end{array}$ & Yes & $\begin{array}{l}\text { Persistent } \\
\text { infection }\end{array}$ & \multirow[t]{2}{*}{ Yes" } \\
\hline & CVC infection & Not treated & N/A & Recurrent infection & \\
\hline 4 & VAP & $\begin{array}{l}\text { Empiric cefepime for } 3 \text { days, } \\
\text { empiric imipenem for } 4 \text { days }\end{array}$ & Yes & Single episode & Yes $^{\|}$ \\
\hline 5 & $\begin{array}{l}\text { Respiratory tract } \\
\text { colonization }\end{array}$ & N/A & N/A & $\mathrm{N} / \mathrm{A}$ & Yes \\
\hline \multirow[t]{2}{*}{6} & CVC infection & $\begin{array}{l}\text { Not treated. The patient } \\
\text { was receiving treatment } \\
\text { for Salmonella and Enterococcus } \\
\text { species sepsis with ampicillin } \\
\text { and gentamicin }\end{array}$ & $\mathrm{N} / \mathrm{A}$ & $\begin{array}{l}\text { Persistent } \\
\text { infection }\end{array}$ & \multirow[t]{2}{*}{ Yes" } \\
\hline & UTI & $\begin{array}{c}\text { Empiric imipenem } \\
\text { for } 2 \text { days, empiric cefepime } \\
\text { for } 1 \text { day }\end{array}$ & Yes & - & \\
\hline \multirow[t]{2}{*}{7} & CVC sepsis & Cefepime for 15 days & Yes & Persistent infection & \\
\hline & Septicemia & $\begin{array}{l}\text { Piperacillin-tazobactam } \\
\text { for } 4 \text { days }\end{array}$ & Yes & - & Yes \\
\hline \multirow[t]{2}{*}{8} & CVC sepsis & $\begin{array}{c}\text { Cefepime for } 8 \text { days } \\
\text { for febrile neutropenia, } \\
\text { MDR-PA growth in blood, } \\
\text { had CVC removed and then } \\
\text { received cefepime for } 14 \text { days, } \\
\text { piperacillin-tazobactam for } \\
11 \text { days, and piperacillin-tazobactam } \\
\text { and levofloxacin for } 14 \text { days }\end{array}$ & No & Single episode & No \\
\hline & Septicemia & Empiric cefepime for 1 day & $\mathrm{N} / \mathrm{A}$ & Recurrent infection & Yes" \\
\hline 9 & Urinary sepsis & $\begin{array}{l}\text { Empiric imipenem for } 2 \text { days, } \\
\text { cefepime for } 9 \text { days }\end{array}$ & Yes & Single episode & Yes \\
\hline \multirow[t]{2}{*}{10} & UTI & $\begin{array}{l}\text { Cefepime for } 22 \text { days, } \\
\text { transferred to another hospital } \\
\text { for J ureteral stent insertion }\end{array}$ & No & Single episode & No \\
\hline & UTI & Polymyxin B for 16 days & $\begin{array}{l}\text { Not } \\
\text { determined }\end{array}$ & $\begin{array}{l}\text { Recurrent } \\
\text { infection }\end{array}$ & Yes \\
\hline
\end{tabular}


Table 2. Clinical presentations, antibiotic regimen, and outcomes in patients with MR-PA infection or colonization*

\begin{tabular}{|c|c|c|c|c|c|}
\hline Patient & $\begin{array}{l}\text { Type of infection/ } \\
\text { colonization }\end{array}$ & $\begin{array}{l}\text { Antibiotic } \\
\text { regimen }^{\dagger}\end{array}$ & $\begin{array}{c}\text { Treatment } \\
\text { failure }^{\ddagger}\end{array}$ & $\begin{array}{c}\text { Infection } \\
\text { classification }^{\S}\end{array}$ & $\begin{array}{l}\text { Death during } \\
\text { hospitalization }\end{array}$ \\
\hline 11 & Urinary sepsis & $\begin{array}{l}\text { Empiric cefepime and } \\
\text { guided by culture for } 14 \text { days }\end{array}$ & Yes & Single episode & Yes \\
\hline 12 & Septicemia & $\begin{array}{c}\text { Empiric piperacillin- } \\
\text { tazobactam for } 7 \text { days, } \\
\text { ciprofloxacin for } 6 \text { days } \\
\text { Cefepime and polymyxin B } \\
\text { for } 3 \text { days, polymyxin B } \\
\text { alone for } 6 \text { days }\end{array}$ & $\mathrm{N} / \mathrm{A}$ & $\begin{array}{l}\text { Persistent } \\
\text { infection }\end{array}$ & No \\
\hline 13 & $\begin{array}{l}\text { Urinary tract } \\
\text { colonization }\end{array}$ & N/A & N/A & N/A & No \\
\hline \multirow[t]{2}{*}{14} & Bacteremia & $\begin{array}{l}\text { Not treated; the patient was } \\
\text { receiving treatment for MDR } K \text {. } \\
\text { pneumoniae CVC sepsis with } \\
\text { piperacillin-tazobactam for } 10 \text { days } \\
\text { and meropenem for } 7 \text { days }\end{array}$ & $\mathrm{N} / \mathrm{A}$ & $\begin{array}{l}\text { Persistent } \\
\text { infection }\end{array}$ & \multirow[t]{2}{*}{ Yes } \\
\hline & VAP & $\begin{array}{l}\text { Empiric cefepime for } 3 \text { days, } \\
\text { polymyxin B for more than } 21 \text { days }\end{array}$ & Yes & - & \\
\hline 15 & $\begin{array}{l}\text { Urinary tract } \\
\text { colonization }\end{array}$ & $\mathrm{N} / \mathrm{A}$ & $\mathrm{N} / \mathrm{A}$ & $\mathrm{N} / \mathrm{A}$ & No \\
\hline 16 & UTI & $\begin{array}{c}\text { Empiric cefepime for } 2 \text { days; } \\
\text { patient transferred to another } \\
\text { hospital }\end{array}$ & $\begin{array}{l}\text { Lost to } \\
\text { follow-up }\end{array}$ & $\begin{array}{l}\text { Lost to } \\
\text { follow-up }\end{array}$ & No \\
\hline
\end{tabular}

CVC, central vascular catheter; N/A, not applicable.

*MR-PA includes the entire resistance pattern (MDR-PA, XDR-PA, and CR-PA).

tDescribed therapy only for Gram-negative bacteria after MR-PA detection.

*Treatment failure: clinical deterioration or persistence of the microorganism in clinical materials resulting in a request to change the antibiotic-based therapy or resulting in death.

\$Infection classification: I) recurrent infection: clinical symptoms of infection reinitiated and isolation of the MR-PA in other or same biologic materials after completion of treatment; II) persistent infection: maintenance of the microorganism in the same or other clinical materials for at least 21 days independent of symptoms or treatment; III) single infection: unique episode of infection with no recurrent or persistent infection.

"Death before last microbiological result with recovery of MR-PA.

Six patients (patient \#1, \#3, \#8 to \#11) with MR-PA infections were treated with cefepime alone or in combination therapy with other antipseudomonal antibiotics considered adequate according to laboratory test results and time of initiation and duration of therapy. However, three of these patients - two with urinary sepsis (patients $\# 9$ and \#11) and one with VAP (patient \#3) - had no responses to 9,14 , and 20 days into antibiotic treatment, respectively. In addition, in patient \#3, MDR-PA regrew in a bronchoalveolar lavage (BAL) sample on the 15th day of treatment with cefepime, and XDR-PA grew in a tracheal aspirate and on a catheter tip on the 4th day after discontinuation of cefepime-based treatment.

\section{Results of culture of environmental samples}

MDR-PA was cultured from 2 of 12 (17\%) environmental samples (water obtained from a humidifier in one patient and a respiratory circuit condensate from the other) collected during the first outbreak in the ICU, which had only two beds at that time. None of the cultures of clinical surfaces, air, or health care workers' hands in the ICU and wards obtained during the second outbreak were positive for P. aeruginosa.

\section{Antibiotic susceptibility profiles and molecular analysis of MR-PA isolates}

All $P$. aeruginosa isolates $(\mathrm{n}=94)$ underwent antibiotic susceptibility testing. Thirty-three isolates from patients 20 MDR-PA, 12 XDR-PA, and 1 CR-PA - had antibiotic- 
resistant profiles (Figure 1). The MDR-PA strains were universally resistant to gentamicin, amikacin, cefotaxime, ticarcillin-clavulanic acid, imipenem, meropenem (tested since January 2005), ciprofloxacin, levofloxacin (tested since September 2004), norfloxacin, and trimethoprim-sulfamethoxazole. Fifteen MR-PA strains isolated from six patients in the first outbreak were susceptible to cefepime, ceftazidime, and polymyxin B; one was susceptible to aztreonam; and six were susceptible to piperacillin-tazobactam.

The index case, five of 11 patients in the outbreaks and one patient from the interepidemic period had one MDR-PA or XDR-PA isolate each analyzed by PFGE (Table 1, Figure 2). The two MR-PA-positive environmental strains also underwent PFGE. Figure 2 shows that all of the PFGE-tested strains had identical genetic profiles and belonged to the São Paulo clone (the SPM-1 strain was matched with MR-PA isolates). However, all of these strains were negative for tested MBL production.

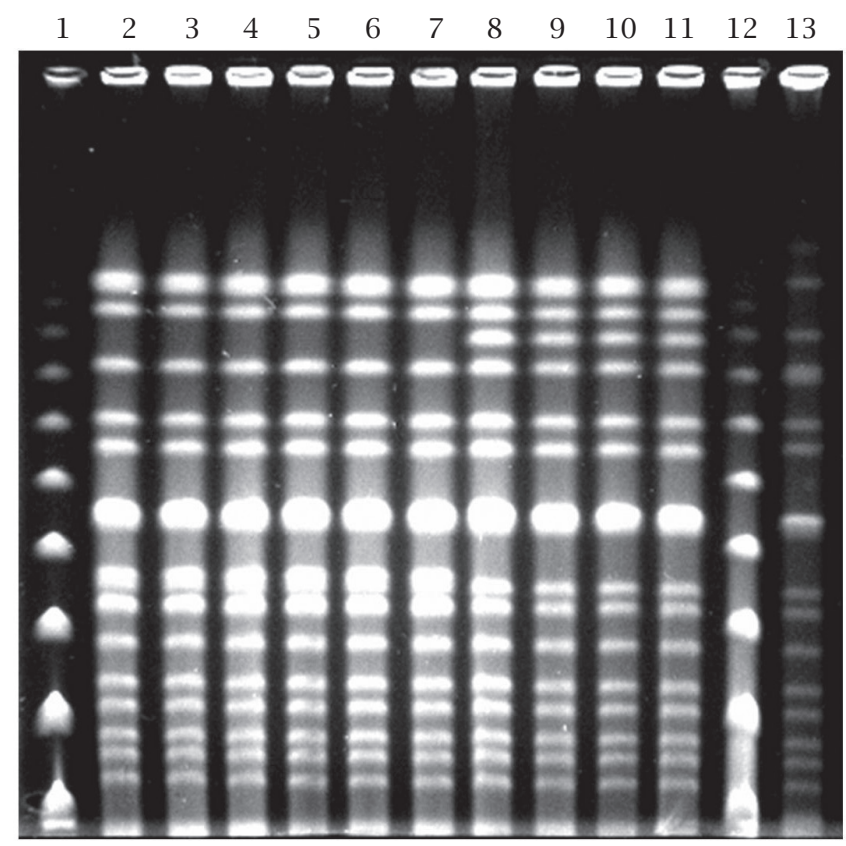

Figure 2: PFGE patterns of 10 isolates of MDR-PA and XDR-PA. Lanes 1 and 12, PFGE Lambda (molecular markers). Lanes 2-11, PFGE-detected patterns in 10 strains of MDRPA or XDR-PA: 2, XDR-PA, patient 1 (index case), blood; 3, XDR-PA, patient 2 ( $1^{\circ}$ outbreak), BAL; 4 , MDR-PA, patient 3 , tracheal aspirate ( $1^{\circ}$ outbreak); 5 and 6 , MDR-PA, swab from condensate of the ventilator circuit of patient $2\left(1^{\circ}\right.$ outbreak); 7, MDR-PA, water sample from a humidifier ( $1^{\circ}$ outbreak); 8 , XDR-PA, patient 6 , urine (non-outbreak case); 9, XDR-PA, patient 7 , urine ( $2^{\circ}$ outbreak); 10 , XDRPA, patient 8 , blood ( $2^{\circ}$ outbreak); 11 , XDR-PA, patient 9 , blood ( $2^{\circ}$ outbreak). Lane 13, São Paulo MLB strain.

\section{Infection control intervention and education program}

Infection-control measures involved outbreak notification, cohort of patients and professionals, temporary closing of the ICU to new admissions during the first outbreak, contact precautions for all colonized and/or infected patients, environmental disinfection, and a review of all disinfection and sterilization processes. During the first outbreak, the infection control team reviewed the health care providers' work process to minimize cross-transmission of MR-PA, including technicians involved in performing hemodialysis from a contracted service. Hospital personnel repaired a water leak in the compressed air system and changed the air conditioning filters in the ICU. Furthermore, the hospitals newly constructed ICU opened in June 2006. Members of the infection control committee systematically presented the surveillance results, including results of molecular analysis of the $P$. aeruginosa strains and the patients' outcomes, to the hospital staff in educational activities carried out over the entire study period to ensure their commitment to infection control measures. However, the hospitals' nursing staff underwent a great turnover in March 2005, whereas the physician and nursing staff did so in November 2006.

\section{DISCUSSION}

Researchers have demonstrated the difficulty of controlling MR-PA infections in hospitals where the agent is already endemic. ${ }^{21}$ However, a multifaceted infection control program can temporarily control the transmission of this infection. ${ }^{3,5}$ To the best of our knowledge, this is the first report to describe the epidemiological characteristics of MDR-PA/XDRPA infection outbreaks that were temporarily controlled but not eradicated in patients with AIDS, in whom persistent MR-PA infections likely increase the risk of transmission.

The reported overall incidence of $P$. aeruginosa carriage in ICUs and oncological/hematological or medical wards has varied from $11.7 \%$ to $37.0 \%{ }^{22,23}$ Many authors have advocated the use of surveillance cultures to improve the control of infections with multiresistant pathogens in high-risk settings, but the benefit of this strategy remains to be evaluated. ${ }^{24}$ We agree with previous observation that surveillance of patients with MR-PA infections must be accompanied by an early implementation of an intervention program whenever required. ${ }^{3}$ In the present study, we based the surveillance program on clinical microbiological examinations of patients' samples requested by infectious disease specialists, who usually have high diagnostic suspicion of nosocomial infections. In addition, we systematically used surveillance cultures with rectal swabs during the two outbreaks; however, we used them in a non-systematic manner between outbreaks. We performed rectal swabs only, as respiratory and urinary samples were normally used for diagnosis of infections. In addition, gastrointestinal carriage appears to 
be a prerequisite for $P$. aeruginosa colonization and/or infection at other sites, and the intestinal tract is considered the most important reservoir of $P$. aeruginosa. ${ }^{22,25}$ However, the sensitivity of rectal swabs in detecting small inocula is suboptimal, ${ }^{22}$ and patients with colonization at sites other than the intestinal tract may have been missed..$^{25,26}$ Our intention was to perform surveillance of patients with MR-PA infections in a real-life hospital setting with limited resources so that the surveillance program would be easy to accomplish. This may have been a limitation of our study, though, as well as our lack of use of selective antimicrobial media for rectal swabs to detect antibiotic-resistant $P$. aeruginosa. ${ }^{23}$

Patients with MR-PA colonization and/or infections frequently have contamination of their hands, clothes, and/ or immediate surroundings, which raises the possibility of transmission to other patients either directly or via contact with hospital staff or contaminated equipment. ${ }^{1,2,6,27}$ During the first outbreak, four patients were undergoing hemodialysis performed by the same technician. We controlled this epidemic after we reviewed this technician's work and assigned different technicians for each patient. Nevertheless, we did not obtain cultures from the hands of the original technician. This horizontal transmission of MR-PA infections was supported by the findings of genotyping analysis of MR-PA isolates obtained from patients and environmental and of the temporal and spatial association among MR-PA cases.

As previously described, we obtained samples of water and wet surfaces contaminated with P. aeruginosa in our surveillance report only in close proximity to infected/colonized patients. ${ }^{3,27}$ Studies showed that airborne dissemination can have a role in the transmission of $P$. aeruginosa. ${ }^{27,28}$ However, we did not detect any $P$. aeruginosa isolates using an air sampler that has equivalent results of the sixth stage of an Andersen air sampler. ${ }^{29}$ The small number of air samples obtained in our study may have influenced this result. The newly renovated ICU in our hospital (with isolation rooms and a modern ventilation system) may have contributed to temporary control of the infection, as suboptimal ICU designs layouts such as our previous one have been associated with MR-PA infection outbreaks. ${ }^{4}$ The organism's panantibioticresistance pattern and biofilm formation most likely played important roles in extending the outbreaks. ${ }^{2}$ Also, hospital personnel shortage ${ }^{30}$ or, as in our case, high hospital staff turnover may be related to MR-PA infection outbreaks or initial difficulty in controlling them. In addition, the second outbreak may have been related to readmission of an MR-PA colonized patient which was likely colonized during hospitalization at the time of the first outbreak; however, we cannot rule out other explanations not fully explored in this study.

The continuous training program on prevention and control of nosocomial infections given to all health care professional categories was an essential tool for controlling
MR-PA infections in our hospital, as well. ${ }^{5,12}$ We used several educational activities and resources such as posters, folders, and signs during the study period. Continuous education is important even in hospitals specialized in treating infectious diseases, where all health care workers have some knowledge about the risk of nosocomial infections. Keeping the clinical staff informed about the surveillance results, clinical outcomes of infected patients, and, when feasible, molecular characteristics of isolated strains is essential to increase and maintain their commitment to controlling nosocomial infection. This is especially important for high-risk populations while new preventive and treatment strategies for MRPA infections are being developed.

We observed some differences in the antibiotic susceptibility patterns between the two outbreaks, although the genetic profiles of the tested strains were identical. The antibiotic-resistance patterns of MR-PA isolates changed during the five-year study period, with MR-PA strains becoming resistant to almost all of the antibiotics tested except polymyxin B. Six patients had P. aeruginosa isolates susceptible or less resistant to the antibiotics tested prior to detection of more antibiotic-resistant strains. All of these patients had received cefepime alone or in combination therapy before isolation of $P$. aeruginosa strains with the patterns of greater antibiotic resistance. Therefore, although cross-colonization was an important feature of these outbreaks, antimicrobial pressure was also likely responsible for these differences in antibiotic resistance. However, in settings of infrequent antimicrobial use, frequent cross-transmission events can contribute to high antibiotic resistance rates. ${ }^{31}$ We did not completely assess the resistance mechanism in our tested strains, but all of the isolates were negative for MBL production. Unfortunately, we did not have the necessary resources to study other antibiotic-resistance mechanisms previously described in MR-PA. ${ }^{8,32}$ MDR-PA infections probably acquired new or derepressed resistance mechanisms during the study period. Sequential emergence of resistance is likely due to the administration of different antibiotics at different time periods following the development of resistance. ${ }^{33}$

The high mortality rates in this study demonstrated the burden of MR-PA infections in immunocompromised patients. This may have reflected the immunocompromised states of the patients as well as the multidrug resistance and possible enhanced virulence of the outbreak strain. ${ }^{2}$ All patients who died had severe comorbidities or opportunistic diseases that likely contributed to the high mortality rates. Inappropriate empirical antimicrobial-based therapy and delays to initiate appropriate antimicrobial-based therapy are both detrimental to patient outcome. ${ }^{34}$ Authors have described failure of cefepime-susceptibility breakpoints to predict clinical outcomes $^{35}$ as well as the requirement of achieving adequate drug exposure to be successful in treating patients with 
P. aeruginosa infections. ${ }^{36}$ Additionally, a multivariate logistic regression analysis demonstrated $P$. aeruginosa infection to be independently associated with treatment failure in cefepime-patients. ${ }^{37}$ We did not evaluate any of these features in the present study; therefore, we cannot attribute the high mortality rates to the antibiotics used.

Investigators have observed relapsed or recurrent infections caused by non-multiresistant $P$. aeruginosa in patients with AIDS but without any impact on mortality. ${ }^{9}$ In addition, authors have described persistent respiratory infections with sequentially obtained non-multiresistant $P$. aeruginosa strains in AIDS patients. ${ }^{10}$ The incidence of treatment failure and persistent or recurrent infections was markedly elevated in the present study. Also, persistent and recurrent infections were observed in patients with and without AIDS. The criteria used for identification of persistent and recurrent infections were based on clinical and antibiotic susceptibility data but not the genetic profiles of the isolated strains, as we did not perform molecular typing of all of the MR-PA isolates (only of randomly preserved strains). In addition, we obtained rectal swabs only from patients who were negative for MR-PA in close contact with a MR-PA positive patient. Consequently, we did not investigate colonization of $P$. aeruginosa in the gastrointestinal tract of patients with MR-PA infections or colonization at other sites. The investigation of MR-PA colonization in the gastrointestinal tract may contribute to understanding the characteristics of persistent and/or recurrent infections caused by MR-PA in these patients. Therefore, more investigations of persistent infections caused by $P$. aeruginosa are necessary in this era of antimicrobial resistance in patients other than those with cystic fibrosis in whom this feature is well recognized. ${ }^{38,39}$

Regarding patients with symptomatic HTLV-1 infections, studies have suggested that they are at increased risk for UTIs. ${ }^{40}$ Physicians have used prolonged urinary catheterization in these patients because of their myelopathy and/or tropical spastic paraparesis. Urinary catheterization and UTIs are both recognized risk factors for MR-PA infection. ${ }^{41,42}$

\section{CONCLUSIONS}

A multifaceted infection control program can control and prevent temporarily the transmission of MR-PA infection in the AIDS setting. Broader investigation of patient environments as well as risk factors for MR-PA infection and/or colonization may help increase understanding of the multifactor origin of MR-PA infection and develop local recommendations for prevention and control of the infection. Infection control programs and diagnosis and treatment approaches of MR-PA infections in AIDS patients must consider the persistent characteristic of MR-PA infections in this population.

\section{ACKNOWLEDGEMENTS}

We thank Dimitrios P. Kontoyiannis for useful comments.

\section{REFERENCES}

1. Deplano A, Denis O, Poirel L et al. Molecular characterization of an epidemic clone of panantibiotic-resistant Pseudomonas aeruginosa. J Clin Microbiol 2005; 43:1198-204.

2. Yakupogullari Y, Otlu B, Dogukan M et al. Investigation of a nosocomial outbreak by alginate-producing pan-antibioticresistant Pseudomonas aeruginosa. Am J Infect Control 2008; 36:e13-8.

3. Adachi JA, Perego C, Graviss L et al. The role of interventional molecular epidemiology in controlling clonal clusters of multidrug resistant Pseudomonas aeruginosa in critically ill cancer patients. Am J Infect Control 2009; 37:442-6.

4. Hota S, Hirji Z, Stockton K et al. Outbreak of multidrug-resistant Pseudomonas aeruginosa colonization and infection secondary to imperfect intensive care unit room design. Infect Control Hosp Epidemiol 2009; 30:25-33.

5. Cortes JA, Cuervo SI, Urdaneta AM et al. Identifying and controlling a multiresistant Pseudomonas aeruginosa outbreak in a Latin-American cancer centre and its associated risk factors. Braz J Infect Dis 2009; 13:99-103.

6. Cezário RC, Duarte De Morais L, Ferreira JC et al. Nosocomial outbreak by imipenem-resistant metallo-beta-lactamaseproducing Pseudomonas aeruginosa in an adult intensive care unit in a Brazilian teaching hospital. Enferm Infecc Microbiol Clin 2009; 27:269-74.

7. Samuelsen O, Toleman MA, Sundsfjord A et al. Molecular epidemiology of metallo-beta-lactamase-producing Pseudomonas aeruginosa isolates from Norway and Sweden shows import of international clones and local clonal expansion. Antimicrob Agents Chemother 2010; 54:346-52.

8. Zavascki AP, Carvalhaes CG, Picão RC, Gales A.C. Multidrugresistant Pseudomonas aeruginosa and Acinetobacter baumannii: resistance mechanisms and implications for therapy. Expert Rev Anti Infect Ther 2010; 8:71-93.

9. Domingo P, Ferré A, Baraldès MA et al. Pseudomonas aeruginosa bronchopulmonary infection in patients with AIDS, with emphasis on relapsing infection. Eur Respir J 1998; 12:107-12.

10. Asboe D, Gant V, Aucken HM et al. Persistence of Pseudomonas aeruginosa strains in respiratory infection in AIDS patients. AIDS 1998; 12:1771-5.

11. Johnson JK, Arduino SM, Stine OC et al. Multilocus sequence typing compared to pulsed-field gel electrophoresis for molecular typing of Pseudomonas aeruginosa. J Clin Microbiol 2007; 45:3707-12.

12. Pereira GH, Levin AS, Oliveira HB, Moretti ML. Controlling the clonal spread of Pseudomonas aeruginosa infection. Infect Control Hosp Epidemiol 2008; 29:549-52.

13. Garner JS, Jarvis WR, Emori TG et al. CDC definitions for nosocomial infections. Am J Infect Control 1988; 16:128-40.

14. Emori TG, Culver DH, Horan TC et al. National Nosocomial Infections Surveillance system (NNIS): description of surveillance methods. Am J Infect Control 1991; 19:19-35.

15. Horan TC, Andrus M, Dudeck MA. CDC/NHSN surveillance definition of health care-associated infection and criteria for specific types of infections in the acute care setting. Am J Infect Control 2008; 36:309-32.

16. Laing RB. Nosocomial infections in patients with HIV disease. J Hosp Infect 1999; 43:179-85. 
17. Clinical and Laboratory Standards Institute. Performance standards for antimicrobial susceptibility testing. 18th informational supplement. M100-S17. Wayne, PA: NCCLS, 2007.

18. Arakawa Y, Shibata N, Shibayama K et al. Convenient test for screening metallo-beta-lactamase-producing gram-negative bacteria by using thiol compounds. J Clin Microbiol 2000; 38:40-3.

19. Romão CMCPA, De Faria YN, Pereira LR et al. Susceptibility of clinical isolates of multiresistant Pseudomonas aeruginosa to a hospital disinfectant and molecular typing. Mem Inst Oswaldo Cruz 2005; 100:541-8.

20. Tenover FC, Arbeit RD, Goering RV et al. Interpreting chromosomal DNA restriction patterns produced by pulsed-field gel electrophoresis: criteria for bacterial strain typing. J Clin Microbiol 1995; 33:2233-9.

21. Furtado GH, Martins ST, Machado AM et al. Clinical culture surveillance of carbapenem-resistant Pseudomonas aeruginosa and Acinetobacter species in a teaching hospital in Sao Paulo, Brazil: a 7-year study. Infect Control Hosp Epidemiol 2006; 27:1270-3.

22. Thuong M, Arvaniti K, Ruimy R et al. Epidemiology of Pseudomonas aeruginosa and risk factors for carriage acquisition in an intensive care unit. J Hosp Infect 2003; 53:274-82.

23. Lepelletier D, Cady A, Caroff $\mathrm{N}$ et al. Imipenem-resistant Pseudomonas aeruginosa gastrointestinal carriage among hospitalized patients: risk factors and resistance mechanisms. Diagn. Microbiol. Infect. Dis. 2010; 66:1-6.

24. Slekovec C, Navellou JC, Blasco G et al. Is surveillance of Pseudomonas aeruginosa carriage in intensive care units useful? Ann Fr Anesth Reanim 2010; 29:279-82.

25. Bertrand X, Thouverez M, Talon D et al. Endemicity, molecular diversity and colonisation routes of Pseudomonas aerugino$s a$ in intensive care units. Intensive Care Med 2001; 27:1263-8.

26. Fortaleza CM, Figueiredo LC, Beraldo CC et al. Risk factors of oropharyngeal carriage of Pseudomonas aeruginosa among patients from a Medical-Surgical Intensive Care Unit. Braz J Infect Dis. 2009; 13:173-6.

27. Panagea S, Winstanley C, Walshaw M.J et al. Environmental contamination with an epidemic strain of Pseudomonas aeruginosa in a Liverpool cystic fibrosis centre, and study of its survival on dry surfaces. J Hosp Infect 2005; 59:102-7.

28. Clifton IJ, Fletcher LA, Beggs CB et al. A laminar flow model of aerosol survival of epidemic and non-epidemic strains of $\mathrm{Pseu}$ domonas aeruginosa isolated from people with cystic fibrosis. BMC Microbiol 2008;8:105.

29. Nunes ZG, Martins AS, Altoe AL et al. Indoor air microbiological evaluation of offices, hospitals, industries, and shopping centers. Mem Inst Oswaldo Cruz 2005; 100:351-7.
30. Pagani L, Colinon C, Migliavacca R et al. Nosocomial outbreak caused by multidrug-resistant Pseudomonas aeruginosa producing IMP-13 metallo-beta-lactamase. J Clin Microbiol 2005; 43:3824-8.

31. Jonas D, Meyer E, Schwab F, Grundmann H. Genodiversity of resistant Pseudomonas aeruginosa isolates in relation to antimicrobial usage density and resistance rates in intensive care units. Infect Control Hosp Epidemiol 2008; 29:350-7.

32. Quale J, Bratu S, Gupta J, Landman D. Interplay of efflux system, ampC, and oprD expression in carbapenem resistance of Pseudomonas aeruginosa clinical isolates. Antimicrob Agents Chemother 2006; 50:1633-41.

33. Pai H, Kim J-W, Kim J et al. Carbapenem resistance mechanisms in Pseudomonas aeruginosa clinical isolates. Antimicrob Agents Chemother 2001; 45:480-4.

34. Lodise TP, Miller CD, Graves J et al. Clinical prediction tool to identify patients with Pseudomonas aeruginosa respiratory tract infections at greatest risk for multidrug resistance. Antimicrob Agents Chemother 2007; 51:417-22.

35. Bhat SV, Peleg AY, Lodise TP et al. Failure of current cefepime breakpoints to predict clinical outcomes of bacteremia caused by gram-negative organisms. Antimicrob Agents Chemother 2007; 51:4390-5.

36. Crandon JL, Bulik CC, Kuti JL, Nicolau DP. Clinical pharmacodynamics of cefepime in patients infected with Pseudomonas aeruginosa. Antimicrob Agents Chemother 2010; 54:1111-6.

37. Deal E.N., Micek S.T., Reichley R.M., Ritchie D.J. Effects of an alternative cefepime dosing strategy in pulmonary and bloodstream infections caused by Enterobacter spp, Citrobacter freundii, and Pseudomonas aeruginosa: a single-center, openlabel, prospective, observational study. Clin Ther 2009; 31:299. 310.

38. Davies JC. Pseudomonas aeruginosa in cystic fibrosis: pathogenesis and persistence. Paediatr Respir Rev 2002; 3:128-134.

39. Ferreira AG, Leão RS, Carvalho-Assef AP et al. Influence of biofilm formation in the susceptibility of Pseudomonas aeruginosa from Brazilian patients with cystic fibrosis. APMIS 2010; 118:606-12.

40. Murphy EL, Wang B, Sacher RA et al. Respiratory and urinary tract infections, arthritis, and asthma associated with HTLV-I and HTLV-II infection. Emerg Infect. Dis. 2004; 10:109-116.

41. Zavascki AP, Barth AL, Gaspareto PB et al. Risk factors for nosocomial infections due to Pseudomonas aeruginosa producing metallo-beta-lactamase in two tertiary-care teaching hospitals. J. Antimicrob. Chemother 2006; 58:882-5.

42. Peña C, Suarez C, Tubau F et al. Carbapenem-resistant Pseudomonas aeruginosa: factors influencing multidrug-resistant acquisition in non-critically ill patients. Eur. J. Clin. Microbiol. Infect. Dis. 2009; 28:519-22. 